\title{
Simulation model for the analysis of road vehicles global efficiency
}

\author{
G. Boschetti, M. Repetto, L. Damiani \& A. Pini Prato \\ Department of Machinery, Energy Systems and Transportation, \\ University of Genoa, Italy
}

\begin{abstract}
Along with the rapid growth of energy shortage and environmental pollution, the improvement of road vehicles' overall efficiency becomes a topical research argument in automotive areas. A significant contribution towards this goal, apart from modifying the powertrain layout and optimizing engine and mechanical components, could be provided by the development of computer-aided energy management systems.

Vehicular powertrain is a nonlinear dynamic integrated system of electrical, mechanical, chemical and thermodynamic devices, whose primary objective is providing the power needed for motion. In order to analyze such a complex system, a simulation platform for a generic vehicle powertrain has been developed in the Matlab/Simulink environment, on the basis of vehicle characteristics (mass, front area, ...) and loads (friction, route, ...).

The aim of this study is the development of a validated powertrain simulation model, able to test different on-board power and energy management strategies. The paper identifies a series of key parameters useful for the study of the overall vehicle efficiency, highlighting the energy losses associated to each powertrain component and plotting energy flows balance diagram.

The models of the different powertrain components have been characterized and cascade-connected. The powertrain operational logics has been implemented in a control system including power distribution and advanced energy management strategies (e.g. overdrive, Stop\&Start system, regenerative braking).

The simulation model has been validated with New European Driving Cycle literature data and experimental road tests.

Keywords: road vehicle, energy saving, control, simulation model, experimental validation.
\end{abstract}




\section{Introduction}

Although the recent evolution of road vehicles propulsion systems has lead to relevant performance and comfort levels, the necessity of considering objectives connected to aspects such as mobility, energy saving and emissions reduction has revealed some conception limits of the current powertrains.

In the field of trucking, energy is utilized with very low conversion efficiencies, due to the existence of a great number of small plants (vehicles) each equipped with a small energy converter (the engine), whose operation is not optimized for most of the driving conditions imposed by the different routes. The research has recently turned its attention towards innovative propulsion systems, able to overtake the problems connected to polluting emissions and fuel consumption of traditional systems, yet paying attention to aspects such as drivability, safety, reliability and performances, that, if neglected, could determine the commercial failure of an innovative solution.

The role that these technologies may play on the world energy balance has been enlightened in several works [1-3]. Transports area is responsible for about $18 \%$ of the world energy consumption and over $57 \%$ of the petroleum fuels consumption. These last, in fact, cover $95 \%$ of the $22 \times 10^{6} \mathrm{GWh}$ used for people and goods transports in the planet and more than $80 \%$ of this energy is used for road transports, of which $50 \%$ for people and $30 \%$ for goods [1]. The research on alternative vehicles has taken three different directions:

1. use of low impact fuels;

2. local air pollution reduction (delocalization of pollution sources, e.g. electric and hydrogen vehicles);

3. rationalization of vehicles powertrain.

The present work focuses on the study of powertrain rationalization, and in particular on the analysis of the energy flows involved in vehicle powertrains. The increase of conventional vehicles efficiency may be obtained through modifications of the traditional powertrain conceived for traction, operating substantially on two levels:

- powertrain structure, architecture and components;

- control systems, especially those turned to the energy flows managements.

For this purpose, the acquisition of adequate instruments aimed to the energetic study of vehicular powertrains is fundamental, to allow the evaluation of the potential improvements coming from the modifications introduced.

This paper describes the development and the validation of a powertrain dynamic simulator implemented in the Matlab-Simulink environment, able to reproduce the behaviour of vehicular powertrains at the different conditions encountered in typical missions.

\section{Energetic analysis of the vehicular powertrain}

In view of automotive powertrain rationalization, it is suitable to consider the vehicles as energy conversion chains, in order to better evaluate the energy flows and the related efficiencies. 
The vehicular resistance to motion (i.e. aerodynamic drag and rolling resistance) calculation represents the starting point of the powertrain energetic study: each contribution to the resistance can in fact be determined exclusively on the basis of vehicle parameters and characteristics of the reference mission chosen. The powertrain energy flows analysis allows to quantify the chain efficiency variation in function of the different missions and powertrain control strategies. The peak efficiency of an internal combustion Diesel engine is close to $40 \%$ and the efficiency of the transmission (i.e. gearbox and differential gear) is around $90 \%$. Therefore, in the automotive field, one should expect global efficiency values higher than $35 \%$. In reality, the values measured for an automobile mean utilization are about $10 \%$ to $15 \%$. The global powertrain efficiency is defined as:

$$
\eta_{g l o b a l}=\frac{\text { Motion Resistance Energy }}{\text { Primary Fuel Energy }}
$$

The present definition only considers as a useful effect the energy needed for winning the resistance to motion, as all the other energies (i.e. potential and kinetic) are conservative and can be, in theory, completely recovered. The global efficiency can also be expressed as:

$$
\eta_{g l o b a l}=\gamma \cdot \eta^{*} \cdot \frac{1-R}{1+\phi}
$$

where:

$$
\gamma=\frac{\bar{\eta}}{\eta^{*}}
$$

represents the "efficiency derating factor"I due to the limited utilization of peak engine power, defined as the ratio between $\bar{\eta}$ (mean efficiency in the engine life) and $\eta^{*}$ (design point efficiency). In eqn (2) $R$ represents the "reverse energy ratio"'i, defined as the ratio between negative and positive energies exchanged between vehicle and road:

$$
R=\frac{\left|W_{r}\right|}{W_{d}}
$$

where $W_{d}$ e $W_{r}$ represent respectively the direct (i.e. from wheels to road) and reverse (i.e. from road to wheels) energies. Finally, $\phi$ defines the ratio between the consumption at the idle condition $F_{0}$ and the consumption during positive energy supply $F^{+}$:

$$
\phi=\frac{F^{0}}{F^{+}}
$$

It is thus possible to estimate the vehicle global efficiency on the basis of the described parameters values. These last depend on the mission typology, i.e.: route (ramps, curves, obstacles, traffic lights, ...); average speed; driving style; engine idling time. In urban and suburban use, typical values of the above mentioned 
parameters may be:

$$
R=0.3 ; \quad \phi=0.1 ; \quad \gamma=\eta^{*}=0.35
$$

from which results:

$$
\eta_{\text {global }}=0.35 \cdot 0.35 \cdot \frac{1-0.3}{1+0.1} \cong 0.08
$$

In order to effectuate an energy analysis able to enlighten the dependence of the above defined parameters on the boundary conditions imposed by the vehicle and the route, it is necessary to simulate the entire powertrain in order to determine the energy flows paths. From these last, it is possible to isolate the components contributions to losses and to evaluate the potential improvements. The simulation model described in the following section allows this type of analysis, obtaining detailed diagrams of the energy flowing from the fuel tank to the wheels.

\section{The vehicle simulation model}

The simulation model described in this paper, implemented in the Matlab / Simulink environment, represents the powertrain of a generic road vehicle. The model can be successively characterized time by time on the specific vehicle to be simulated, providing as model inputs parameters such as frontal area, mass, drag coefficient, engine characteristic curves control logics parameters, ....

The main model input quantities are vehicle instantaneous speed (time law) and slope of the road, parameters characterizing the mission profile imposed to the vehicle. As output, the model provides the parameters necessary for the vehicle energetic study, the instantaneous value of resistance to motion components and the fuel consumption. The model can also calculate the efficiency of powertrain single components and their contribution to the total losses.

In order to allow a better comprehension and a simpler management of the simulation model modifications, a modular structure with multiple blocks, each representing one component of the powertrain, has been implemented. The different blocks are listed below:

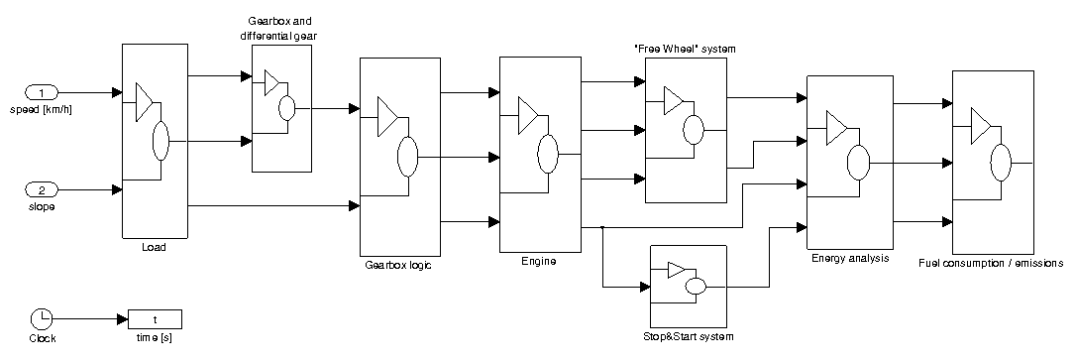

Figure 1: Simulation model main screenshot. 
Load: this block calculates the different contributions to vehicle resistance imposed by mission (tire rolling, aerodynamic resistance, inertia, ramps and drops), and so the power required for motion.

Gearbox and differential gear: in which the energy lost into heat due to friction in the gears is computed.

Gearbox logic: allows to set the gearbox transmission ratio between engine and wheels in function of vehicle speed and power required for motion. The gearbox transmission ratio is imposed on the basis of opportunely determined transmission maps: these last associate to every couple of values (load, vehicle speed) a discrete value of the gearbox transmission ratio. The authors have built different transmission maps, each related to a driving style, in order to take into account the influence of different drivers characteristics on the powertrain efficiency.

Engine: in the paper, the authors have chosen to concentrate their efforts on vehicles equipped with Diesel engines[4]. The Engine block computes the thermal engine internal energy losses, of both mechanic and thermodynamic nature. In the block are implemented efficiency maps in function of engine rotational speed and load required. These maps have been derived from literature experimental data effected on Diesel engines, provided in the form of efficiency values normalized by engine power in order to be representative of the behavior of different size engines. An example of such maps is shown in fig. 2, representing the 3D map of the indicated efficiency. As visible, power is provided as a non-dimensional value, whereas speed is represented in revolutions per minutes: in fact, for the Diesel engines

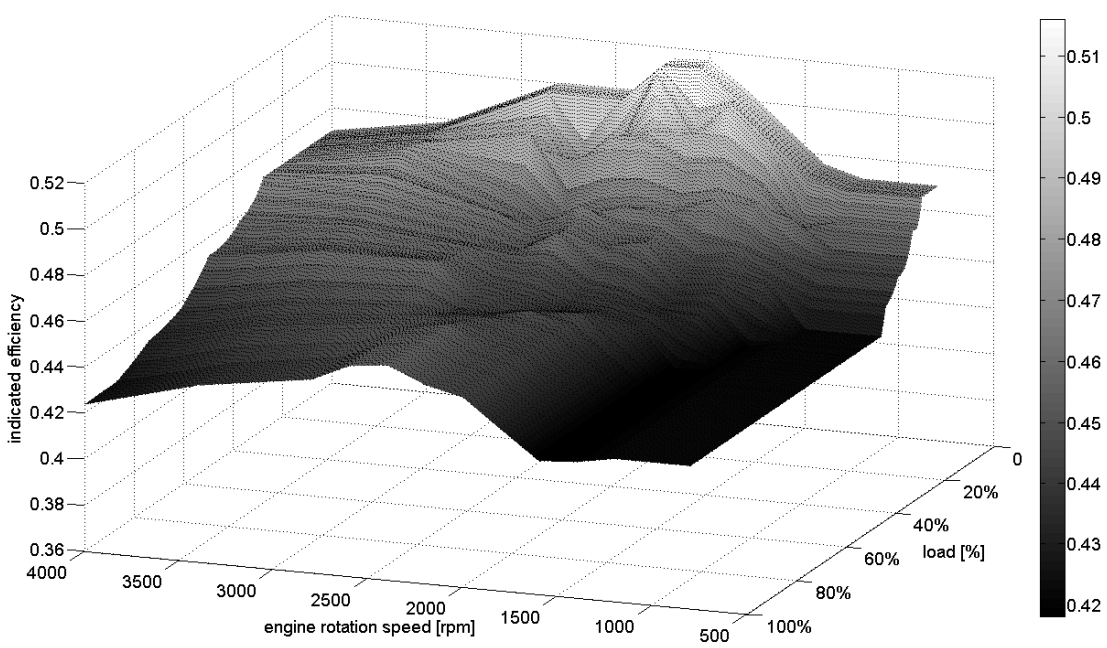

Figure 2: 3D map of the indicated efficiency. 
considered, rotational speed range is substantially independent of the engine size.

Energy analysis: the function of this block is the calculation of the fundamental quantities, defined in section 2, needed for powertrain efficiency and energy flows determination.

Fuel consumption/emissions: these blocks provide the fuel flow rate and pollutants emissions moment by moment.

In order to evaluate possible efficiency-improving modifications to the vehicle control system [5], other blocks implementing different control logics of the powertrain have been predisposed:

- "Stop\&Start" system control: aimed to idle condition consumption limitation;

- regenerative brake: by which is possible the simulation of thermal-electric mild and full hybrid vehicles behavior;

- "Free Wheel" control: aimed to minimizing the losses in the situations of reverse power availability.

\section{Simulation model validation}

In order to make the simulation model a reliable verification tool, it has been validated on the basis of a series of reference missions opportunely chosen. The model output values have been compared with data provided by approval bodies or derived by experimental test drives.

\subsection{The reference vehicle}

The reference vehicle chosen for the simulation model reliability tests is a 2006 Volkswagen Polo 1.4 TDI. This car has been made available by DIMSET for the road tests. Table 1 provides a synthesis of the main characteristics of the chosen vehicle.

\subsection{Routes and reference parameters}

A first verification of the simulation model reliability has been effected taking as reference the New European Driving Cycle (NEDC) data, consisting of four repeated urban (ECE-15) driving cycles and an Extra-Urban (EUDC) driving cycle [6]. Other verifications have been carried out on the basis of experimental data acquired on a urban mission (20 km/h average speed) and on two constant speed (respectively $90 \mathrm{~km} / \mathrm{h}$ and $120 \mathrm{~km} / \mathrm{h}$ ) no slope routes. In particular, the reference urban mission, lasting about 52 minutes, is characterized by a distance of $30 \mathrm{~km}$ and several (40) stop and go.

The model has been validated comparing the simulated fuel consumption with the data declared in the homologation phase for the NEDC cycle and with the data provided by the car on-board instrumentation for the experimental road tests. As 
Table 1: Main characteristics of the reference vehicle.

\begin{tabular}{|lr|}
\hline \multicolumn{3}{|c|}{ Volkswagen Polo 1.4 TDI } \\
\hline \multicolumn{3}{|c|}{ Dimensions \& Weight r } \\
\hline Length & $3916 \mathrm{~mm}$ \\
\hline Width & $1650 \mathrm{~mm}$ \\
\hline Height & $1467 \mathrm{~mm}$ \\
\hline Kerb weight & $1114 \mathrm{~kg}$ \\
\hline Frontal area & $2.04 \mathrm{~m}^{2}$ \\
\hline Fuel capacity & 451 \\
\hline \hline \multicolumn{3}{|c|}{ Engine } \\
\hline Fuel system & direct diesel injection \\
\hline Cylinders & $1422 \mathrm{cc}$ \\
\hline Displacement & (inline) \\
\hline Maximum power & $58.9 \mathrm{~kW} @ 4000 \mathrm{rpm}$ \\
\hline Maximum torque & $195 \mathrm{Nm} @ 2200 \mathrm{rpm}$ \\
\hline \hline & $174 \mathrm{~km} / \mathrm{h}$ \\
\hline Top speed & Performance \\
\hline Fuel consumption & urban/extra-urban/combined \\
\hline
\end{tabular}

a final verification test, the power needed for motion, calculated by the model at vehicle top speed, has been compared with engine declared maximum power: these two power values must tend to equality, since at top speed the engine supplies the maximum power.

\subsection{Results of the validation}

Table 2 reports the comparison between the declared average consumption values and those resulting from the simulations of the reference homologation driving cycles. The presented data put in evidence a substantial correspondence between the simulations results and the consumption values declared for urban route (ECE 15, repeated 4 times), extra-urban (EUDC) and mixed (NEDC) routes. For both

Table 2: Comparison between declared and simulated average consumption values on the reference driving cycles.

\begin{tabular}{|l|c|c|}
\hline route & declared average consumption $\left[\mathbf{y}_{\mathbf{1 0 0}} \mathbf{k m}\right]$ & simulated average consumption $\left[\mathbf{y}_{\mathbf{1 0 0}} \mathbf{k m}\right]$ \\
\hline $\mathrm{ECE}-15 \times 4$ & 5.50 & 5.39 \\
\hline $\mathrm{EUDC}$ & 3.90 & 4.00 \\
\hline $\mathrm{NEDC}$ & 4.40 & 4.45 \\
\hline
\end{tabular}


the routes at constant speed, the average fuel consumptions calculated (about 4.5 $1 / 100 \mathrm{~km} \mathrm{~km}$ at $90 \mathrm{~km} / \mathrm{h}$ and $5.91 / 100 \mathrm{~km}$ at $120 \mathrm{~km} / \mathrm{h}$ ) show a full agreement with the experimental data. The simulation model average fuel consumption calculated for the urban mission, about $5.21 / 100 \mathrm{~km}$, agrees with that measured by the vehicle onboard instrumentation, approximately equal to $5.31 / 100 \mathrm{~km}$. Finally, the verification on maximum power supply is acceptable, as the simulation model indicates a value differing from the homologation data $(58.9 \mathrm{~kW}$ at $4000 \mathrm{rpm})$ of less than $2 \%$.

\section{Discussion of the results}

In table 3 are presented some results obtained in the urban route test drive, in order to enlighten the main potentialities of the simulator. The possibility of isolating the energetic efficiencies and the loss contributions connected to the different powertrain elements, allows to date back to the paths of power and energy flows passing through the powertrain at the different vehicle working conditions. These flows may be visualized by means of Sankey diagrams, as that depicted in fig. 3 .

Table 3 shows the data related to the loss contributions in percentage with respect to the net energy spent for motion (thus, subtracted of the idle condition consumption, equal to circa $5 \%$ of the total fuel consumption).

According to table 3 and to fig. 3, and referring to the definitions provided in section 2 , the following main contributions to the energy flows can be individuated:

Table 3: Energetic analysis results for urban mission.

\begin{tabular}{|c|c|c|c|c|}
\hline Total consumption & {$[\mathrm{kWh}]$} & 12.28 & $100.0 \%$ & \\
\hline Idle consumption & {$[\mathrm{kWh}]$} & 0.57 & $4.6 \%$ & \\
\hline Powertrain input & {$[\mathrm{kWh}]$} & 11.71 & $95.4 \%$ & $100.0 \%$ \\
\hline Thermodynamic losses for positive flywheel power & {$[\mathrm{kWh}]$} & 5.34 & & $45.6 \%$ \\
\hline Thermodynamic losses for negative flywheel power & {$[\mathrm{kWh}]$} & 0.76 & & $6.5 \%$ \\
\hline Thermodynamic losses for zero flywheel power & {$[\mathrm{kWh}]$} & 0.12 & & $1.0 \%$ \\
\hline Mechanical losses for positive flywheel power & {$[\mathrm{kWh}]$} & 2.43 & & $20.8 \%$ \\
\hline Mechanical losses for negative flywheel power & {$[\mathrm{kWh}]$} & 1.38 & & $11.8 \%$ \\
\hline Mechanical losses for zero flywheel power & {$[\mathrm{kWh}]$} & 0.09 & & $0.8 \%$ \\
\hline Transmission losses for direct power & {$[\mathrm{kWh}]$} & 0.09 & & $0.8 \%$ \\
\hline Transmission losses for reverse power & {$[\mathrm{kWh}]$} & 0.04 & & $0.3 \%$ \\
\hline Rolling resistance & {$[\mathrm{kWh}]$} & 0.81 & & $6.9 \%$ \\
\hline Aerodynamic drag & {$[\mathrm{kWh}]$} & 0.41 & & $3.5 \%$ \\
\hline Energy dissipated in the brakes & {$[\mathrm{kWh}]$} & 0.23 & & $2.0 \%$ \\
\hline Average consumption & {$\left[y_{100 \mathrm{~km}]}\right.$} & 5.54 & & \\
\hline Average engine rotation speed & {$[\mathrm{rpm}]$} & 1704.40 & & \\
\hline Engine cycles & [cycles $/ \mathrm{km}$ ] & 5819.47 & & \\
\hline
\end{tabular}




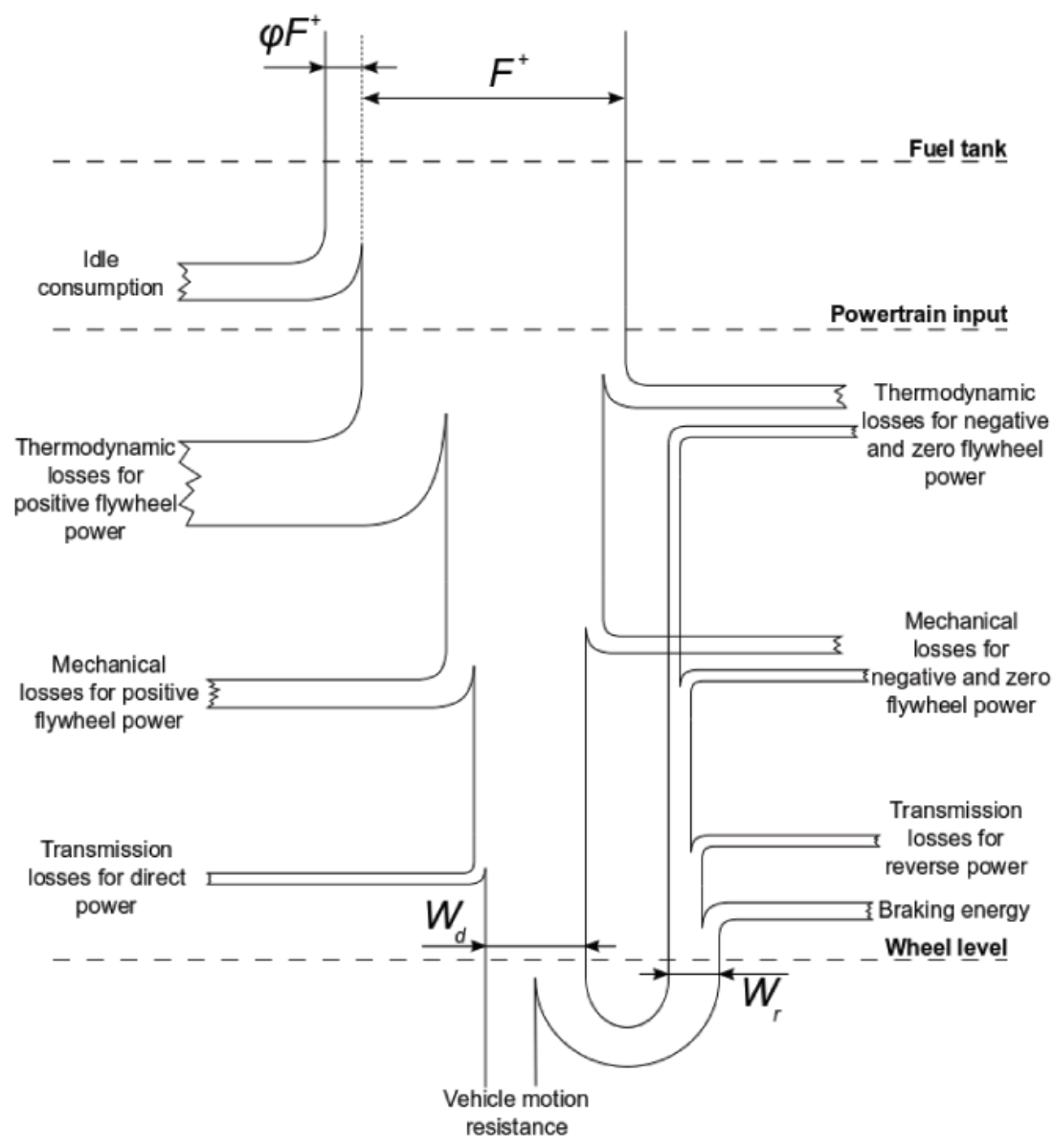

Figure 3: Example of energy flows Sankey diagram.

fuel consumption: the total fuel consumption $(F)$ is divided into the auto-consumption (or idle consumption) $(\phi F)$ and in the fuel utilized for propulsion;

thermodynamic losses: these are the energy losses connected to the thermodynamic cycle operating into the internal combustion engine, classified on the basis of the direction of power at the flywheel (i.e. positive when power flows from the engine to the wheels and negative in the opposite case; the condition of zero power at the flywheel occurs as vehicle is marching with a gear but motion is assured by the conservative forces e.g. road slope. At this condition engine torque, and so power, supplied to the wheels is zero and all the chemical power consumed transforms into losses to keep the engine in rotation at higher revolutions than idle; 
mechanical losses: represent the energy losses connected to internal combustion engine frictions (i.e. piston motion, crank mechanism friction, ...), classified on the basis of the direction of power at the flywheel;

transmission losses: energy losses connected to the efficiency of the transmission devices interposed between engine and wheels, classified on the basis of the direction of power at the flywheel;

friction: these energy losses must be distinguished into the aerodynamic resistance and the rolling resistance of tires. These contributions are purely dissipative and are thus treated separately with respect to the road slope and inertial components, which represent, instead, contributions to conservative energy;

energy dissipated in the brakes: this last component is derived by difference between the energy of the consumed fuel and the energy contributions above defined; it represents the portion of the dissipated conservative energy, and can be considered indicative of the maximum recoverable energy in the chosen duty cycle.

\section{Conclusions}

In the course of the presented work, an analysis of the energy flows related to the private transport road vehicles has been effected with the aid of a simulation model. Despite its apparent specificity, the topic acquires a decisive importance if related to the role played by road transports in the world energy balance.

Notwithstanding the evolution experimented by propulsion systems and the changes in automobile components, the design of road vehicles powertrain has not been interested by radical improvements to date. Moreover, despite a vehicle powertrain efficiency of circa $35 \%$, the life-averaged energy efficiency of the vehicles in circulation is less than $10 \%$. This data puts in evidence the importance of systems aimed to a rational management of powertrain energy flows, having the final goal of global efficiency increase.

A simulation model able to follow the energy flows path for the different driving conditions has been developed; the model is able to isolate each loss contribution of the powertrain components.

The simulation model accuracy has been verified basing on European homologation driving cycles data and on experimental driving tests effected on different routes and missions.

In this way, a very useful tool has been created, easily identifiable with the vehicle tested and able to individuate the saving potentials and verify the effect of possible improvements involving powertrain components and control system.

\section{References}

[1] Organisation for Economic Co-operation Development, International road traffic and accident database (irtad), 2003. 
[2] Institut Français du Pétrole, Energy consumption in the transport sector. Panorama, 2005.

[3] Chan, C., The state of the art of electric, hybrid, and fuel cell vehicles. Proceedings of the IEEE, 95(4), pp. 704-718, 2007.

[4] Shuai, S. \& Youtong, Z., A research on the fuel quantity control for common rail diesel engine in the hybrid electric vehicles working on the low-load area. Industrial Electronics and Applications, 2009. ICIEA 2009. 4th IEEE Conference on, pp. 440-444, 2009.

[5] Boschetti, G., Repetto, M., Damiani, L. \& Pini Prato, A., Road vehicle energy management strategies through a powertrain simulation model, 2010.

[6] Chen, K., Cheng, Y., Bouscayrol, A., Chan, C., Berthon, A. \& Cui, S., Inversion-based control of a hybrid electric vehicle using a split electric variable transmission. Vehicle Power and Propulsion Conference, 2008. VPPC '08. IEEE, pp. 1 -6, 2008. 